\title{
Two's a Company, Three's a Crowd: A Review of Initiating or Switching to a Two-Drug Antiretroviral Regimen in Treatment-Naïve and Treatment- Experienced Patients Living with HIV-1
}

\author{
Melissa Badowski $\cdot$ Sarah E. Pérez $\cdot$ David Silva $\cdot$ Andrea Lee
}

Received: January 27, 2020 / Published online: March 19, 2020

(C) The Author(s) 2020

\begin{abstract}
Introduction: As HIV has become a manageable chronic condition, a renewed and increased interest in challenging traditional three-drug HIV therapies and moving toward two-drug regimens (2DR) for initial or maintenance treatment in people living with HIV (PLWH) has developed. As PLWH are living longer, continual advancements in antiretroviral regimens have been a focus to provide optimal lifelong therapy options. Although early studies may have shown poor outcomes in virologic suppression with $2 \mathrm{DR}$, newer studies and treatment options have emerged to show promise in the management of HIV. The purpose of this review is to evaluate current literature and assess the efficacy of two-drug (2DR) antiretroviral therapy in treatment-naive and -experienced people living with HIV.
\end{abstract}

Digital Features To view digital features for this article go to https://doi.org/10.6084/m9.figshare.11961933.

M. Badowski $(\bowtie) \cdot D$. Silva $\cdot$ A. Lee

College of Pharmacy, University of Illinois, Chicago,

IL, USA

e-mail: badowski@uic.edu

S. E. Pérez

College of Pharmacy, University of New Mexico,

Albuquerque, NM, USA
Methods: A systematic search was performed between January 2009 to January 2020, using EMBASE, MEDLINE, Google Scholar, and bibliographies. Combinations of the following search terms were used: HIV-1 infection, antiretroviral therapy, dual therapy, two-drug regimen, two-drug therapy, two-drug regimen, and 2DR. Included studies were those in the adult population with at least one active comparator, outcomes assessing HIV-1 RNA viral load while on treatment, and written in English. Results: Thirty-three studies were included, 13 where 2DRs were evaluated as initial therapy (3 studies with extension data) and 15 where 2DRs were evaluated as maintenance or switch therapy (2 studies with extension data).

Conclusion: Although 2DRs may not be appropriate in all patient populations, they are being utilized more frequently and have the potential to reduce costs, adverse effects, and drug interactions.

Keywords: Antiretroviral therapy; Dual therapy; HIV; HIV treatment; Two-drug regimen (2DR) 


\section{Key Summary Points}

HIV treatment has evolved from a time where two-drug regimens (2DRs) were once considered a novel concept to current times where they are a reality.

Over the past year, national guidelines recommend 2DRs for initial consideration in the management of HIV for patients meeting certain criteria.

When compared with three-drug regimens, many 2DRs have demonstrated noninferiority, in terms of virologic efficacy, in both the treatment-naïve and experienced populations.

\section{INTRODUCTION}

With continued advancement of antiretroviral therapy (ART), human immunodeficiency virus (HIV) has evolved from an acute and fatal diagnosis to a chronic condition where many patients are able to live a long and healthy life. Although there is currently no cure available for HIV, highly toxic regimens requiring high pill burden and dosing frequency are no longer the standard in care. Antiretroviral medications are now more readily available with durable virologic efficacy, high genetic barriers to resistance, more tolerable side effect profiles, and reduced pill burden. To understand the future of HIV medicine, it is important to review the past lessons learned.

In 1987, the first agent to manage HIV, zidovudine (AZT), was approved as monotherapy in the treatment of people living with HIV (PLWH) [1]. However, the limitations of monotherapy soon became apparent as researchers found the regimen could not sustain virologic suppression and led to the rapid development of drug resistance [1].

In the 1990s, researchers began to shift their focus toward three-drug regimens (3DRs) for the treatment of HIV. This idea ultimately changed and shaped the course of HIV management. In 1996, the combination of a protease inhibitor (PI) with two NRTIs was shown to rapidly reduce HIV RNA levels and improve immune function in patients by targeting different steps within the HIV life cycle [2].

Since the approval of the AZT in 1987, there have been over 30 drugs and 7 different mechanistic drug classes approved for the management of PLWH [3]. Historically, initial ART for treatment-naïve individuals consisted of a twodrug NRTI backbone plus a drug from another drug class such as a non-nucleoside reverse transcriptase inhibitor (NNRTI), integrase strand transfer inhibitor (INSTI), or boosted PI [4]. Several clinical trials and evaluations over the last decade have shown that this three-drug treatment strategy for treatment-naïve patients results in HIV viral suppression and increased immunologic function [4].

The European AIDS Clinical Society (EACS) 2019 guidelines recommend initial regimens in treatment-naïve patients including unboosted INSTI-based regimens, NNRTI-based with rilpivirine (RPV) or doravirine (DOR) or PI-based with boosted darunavir (DRV) [5]. Interestingly, the EACS guidelines were the first to recommend the two-drug regimen (2DR) dolutegravir (DTG)/lamivudine (3TC) for consideration as a recommended regimen in patients whose hepatitis B surface antigen (HBsAg) is negative, HIV1 RNA viral load $<500,000$ copies $/ \mathrm{ml}$, and CD4 $>200$ cells $/ \mathrm{mm}^{3}$ [5]. Shortly thereafter, US-based guidelines also adopted this recommendation.

While 3DRs have been the mainstay of treatment over the last 2 decades, attempts have been made to further reduce medication and pill burden while preventing long-term toxicities and increasing tolerability. Studies have shown that simplifying ART regimens to monotherapy in virologically suppressed PLWH is not a reliable or effective option. In an openlabel, phase 2, randomized non-inferiority trial, DTG monotherapy was non-inferior to combination ART at 24 weeks; however, viriologic failure was seen after 24 weeks with continued monotherapy, which led to DTG resistance [6]. The switch to certain monotherapy treatments occurred in patients already virologically 
suppressed; these regimens have largely failed to maintain viral suppression for an extended duration, with frequent cases of virologic rebound and emerging resistance [6-8].

In November of 2017, DTG/RPV was approved as the first dual ARV single-tablet regimen (STR) for the maintenance therapy of HIV-1 infection [9]. Although DTG/RPV studies have shown efficacy, safety, and tolerability in treatment-experienced patients with sustained virologic suppression, there is still the concern for arising ARV resistance [9]. Overall, DTG/RPV has opened the doors to researching the use of 2DR in in treatment-experienced and -naïve PLWH. Therefore, this article will focus reviewing the efficacy of 2DR as initial or maintenance/switch treatment in PLWH. This article is based on previously conducted studies and does not involve any new studies of human or animal subjects performed by any of the authors.

\section{METHODS}

Manuscripts and abstracts were systematically searched between January 2009 and January 2020, using PubMed, EMBASE, MEDLINE, Google Scholar, conference proceedings, and bibliographies. Combinations of the following search terms were used: HIV-1 infection, antiretroviral therapy, dual therapy, two-drug regimen, two-drug therapy, two-drug regimen, and 2DR. Studies were screened by abstract review for relevance, and only those with at least one active comparator and outcomes assessing HIV-1 RNA viral load while on treatment were included (Fig. 1). In addition, article selection was limited to the English language and adult populations only. Two authors independently replicated the initial literature search to confirm the eligibility of identified studies and screened titles and abstracts of those identified for inclusion in the review with no disagreement.

Information taken from each study included ART regimens, trial design, outcomes including virologic suppression or failure, and the development of resistance. The data from each study were then organized into two main categories of whether patients were treatment naïve or treatment experienced and then further subdivided based on whether they were receiving a regimen that was NRTI inclusive or NRTI sparing.

\section{RESULTS}

\section{Initial HIV Treatment Population}

\section{NRTI Inclusive}

Four randomized, non-inferiority trials were identified of NRTI-inclusive 2DRs in the treatment-naïve population (Table 1) [10-14]. A small study of (lopinavir/ritonavir) LPV/ $\mathrm{r}+$ tenofovir disoproxil fumarate (TDF) vs. $\mathrm{LPV} / \mathrm{r}+$ two non-TDF NRTIs failed to achieve non-inferiority, but was notably underpowered. Unusually high discontinuation rates were also observed among both groups (2DR 42\%; 3DR $44 \%$ ) in addition to poor efficacy (2DR 51\%; 3DR 53\%) [14].

Three studies included lamivudine (3TC) as the single NRTI of their 2DR, with two studies including a boosted PI and one including DTG $[10,12,13]$. High rates of virolologic suppression at 48 weeks were achieved among all three studies, with comparable efficacy shown between groups ranging from 88 to $93 \%$ for 2 DRs vs. $84-94 \%$ for $3 D$ Rs. No cases of treatment-emergent resistance were found among the GEMINI study of DTG + 3TC [10] or the ANDES study of darunavir (DRV)/ $\mathrm{r}+3 \mathrm{TC}$ [12]. Ninety-six-week pooled data from the GEMINI study were recently presented, and non-inferiority was met between 2DR and 3DR [11]. Although 11 people in the 2DR arm and 7 in the 3DR arm met protocol-defined failure, no participant developed treatment-emergent resistance through 96 weeks.

\section{NRTI Sparing}

Of nine non-inferiority studies including a variety of $2 \mathrm{DR}$ combinations, non-inferiority was achieved in five studies [15-18, 24] (Table 1) [15-25]. Two studies included maraviroc (MVC) + boosted PI $[19,20]$. The 2DR of daily $\mathrm{MVC}+\mathrm{DRV} / \mathrm{r}$ was terminated early because of poor comparative efficacy [19]. However, noninferiority was achieved in a subsequent small, 


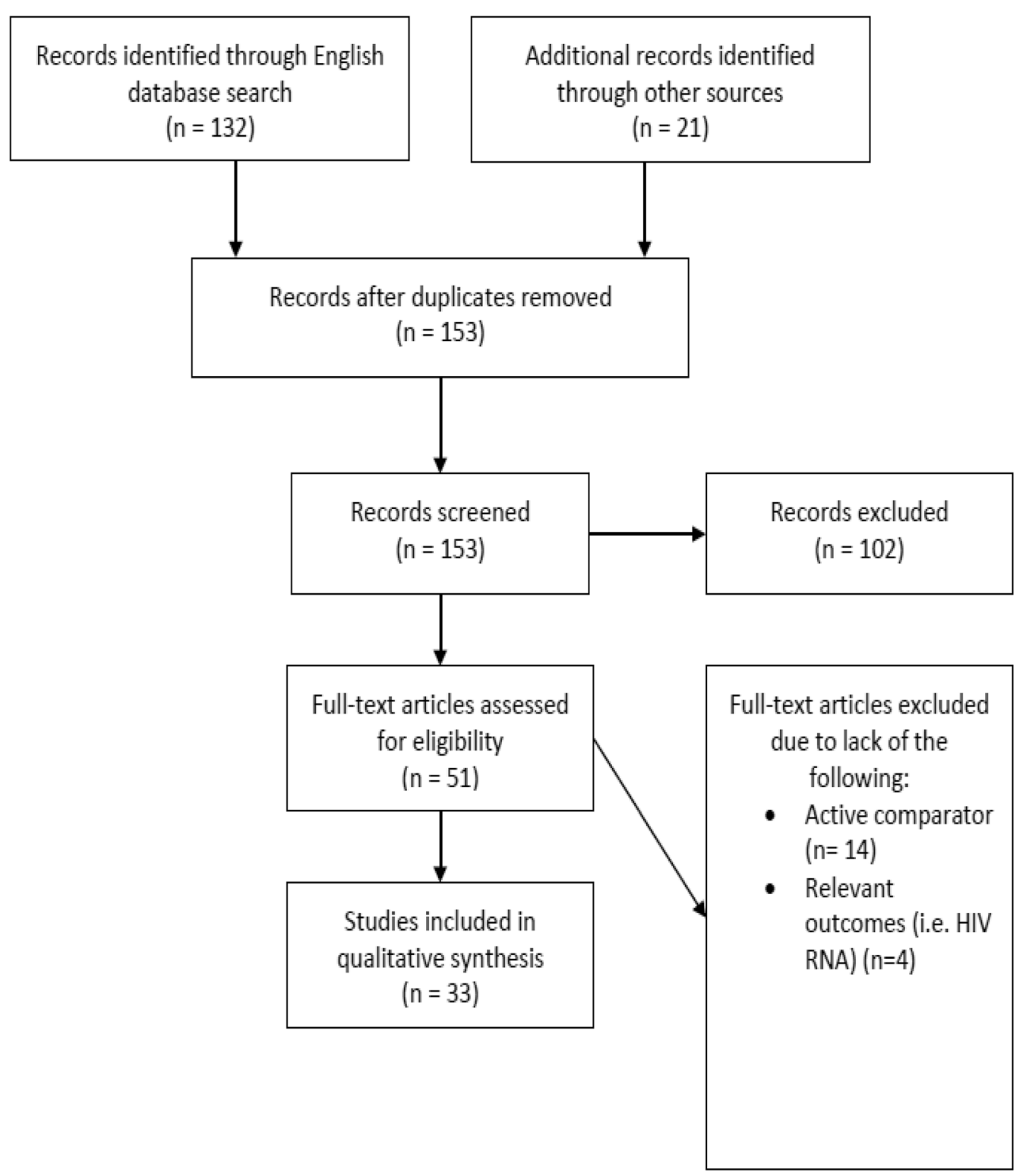

Fig. 1 Study selection process

proof-of-concept study of patients with lower baseline VL (i.e., $<100,000$ copies $/ \mathrm{ml}$ ) initiated on daily $\mathrm{MVC}+$ twice daily $\mathrm{LPV} / \mathrm{r}$ [20]. It should be noted that these findings were not seen in a larger study comparing MVC with a boosted PI in participants with baseline HIV RNA $>100,000$ copies/ml [19]. Although data exist for once-daily maraviroc in treatment-experienced patients with R5 virus, the dose is $300 \mathrm{mg}$ daily instead of $150 \mathrm{mg}$ daily [26]. The lack of the typical $300 \mathrm{mg}$ daily dosing of MVC may have reduced its efficacy and use in this setting.

Three studies included raltegravir (RAL) + boosted PI [23-25]. One study of RAL + LPV/r achieved non-inferiority and reported more favorable changes from the baseline estimated glomerular filtration rate (eGFR) and bone mineral density (BMD) relative to $\mathrm{LPV} / \mathrm{r}+\mathrm{TDF} /$ emtricitabine (FTC) [24]. A small study of DRV/ $\mathrm{r}+\mathrm{RAL}$ vs. DRV/r + TDF/FTC failed to achieve non-inferiority [23]. However, a much larger 


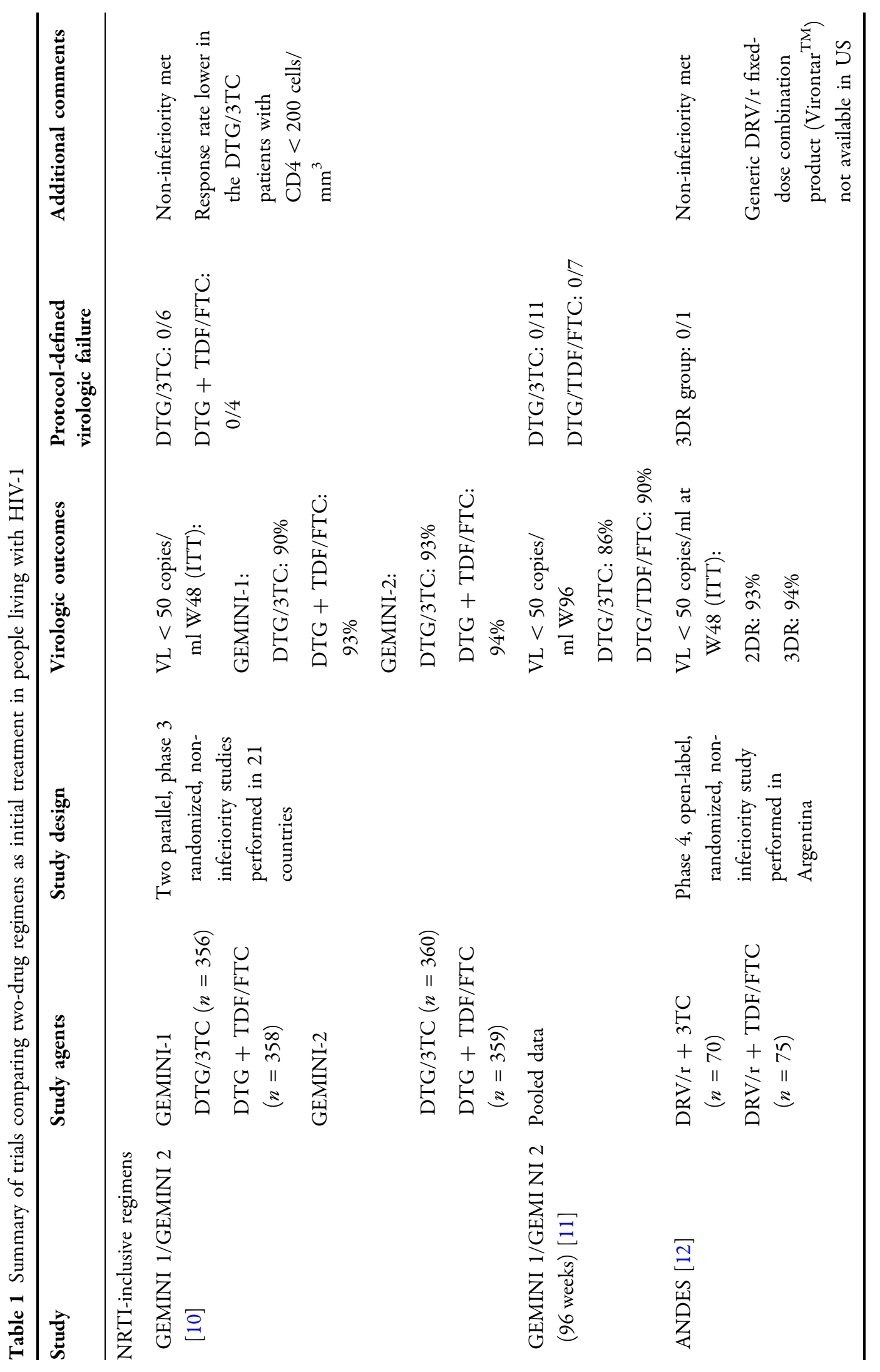




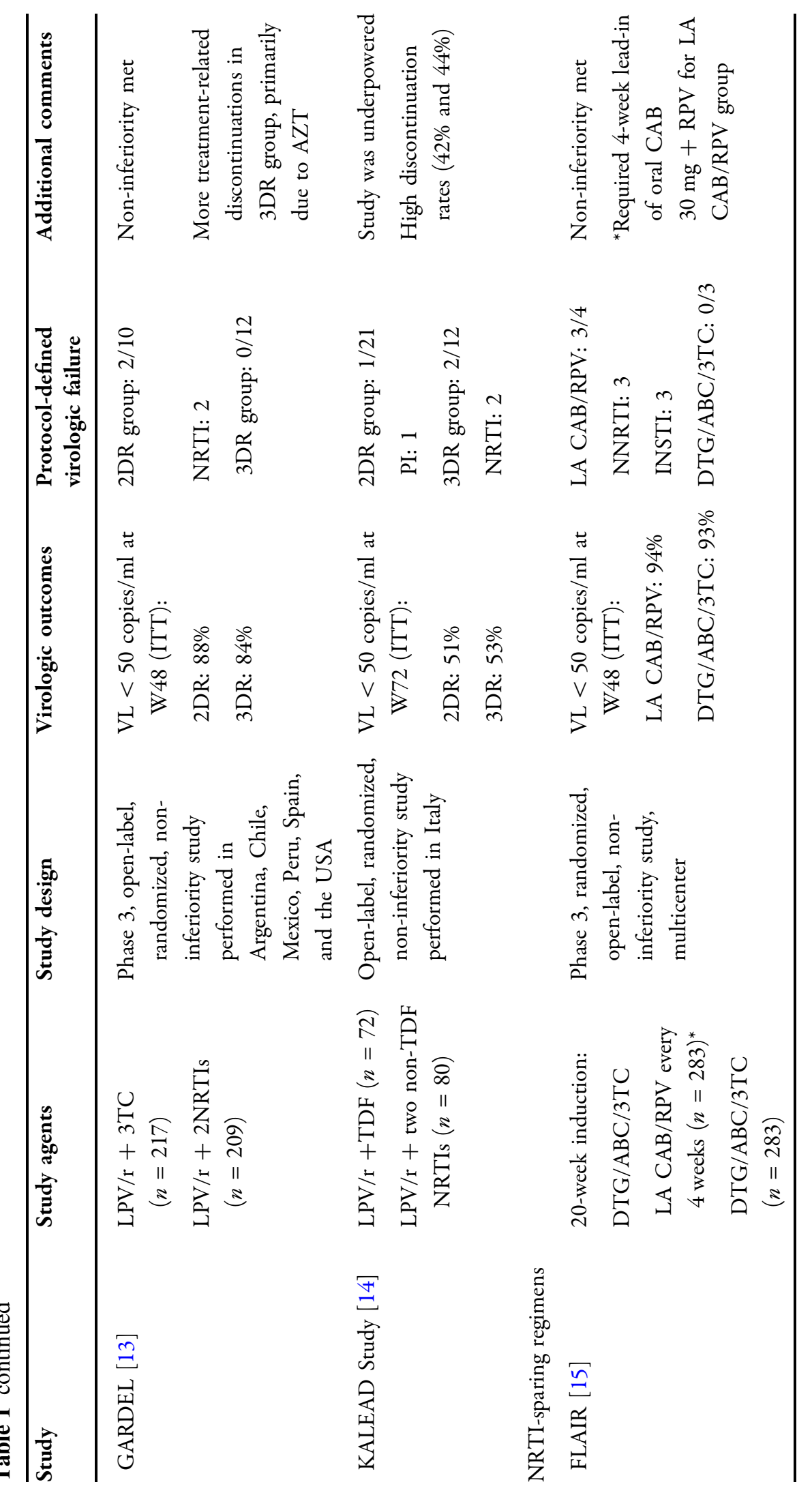




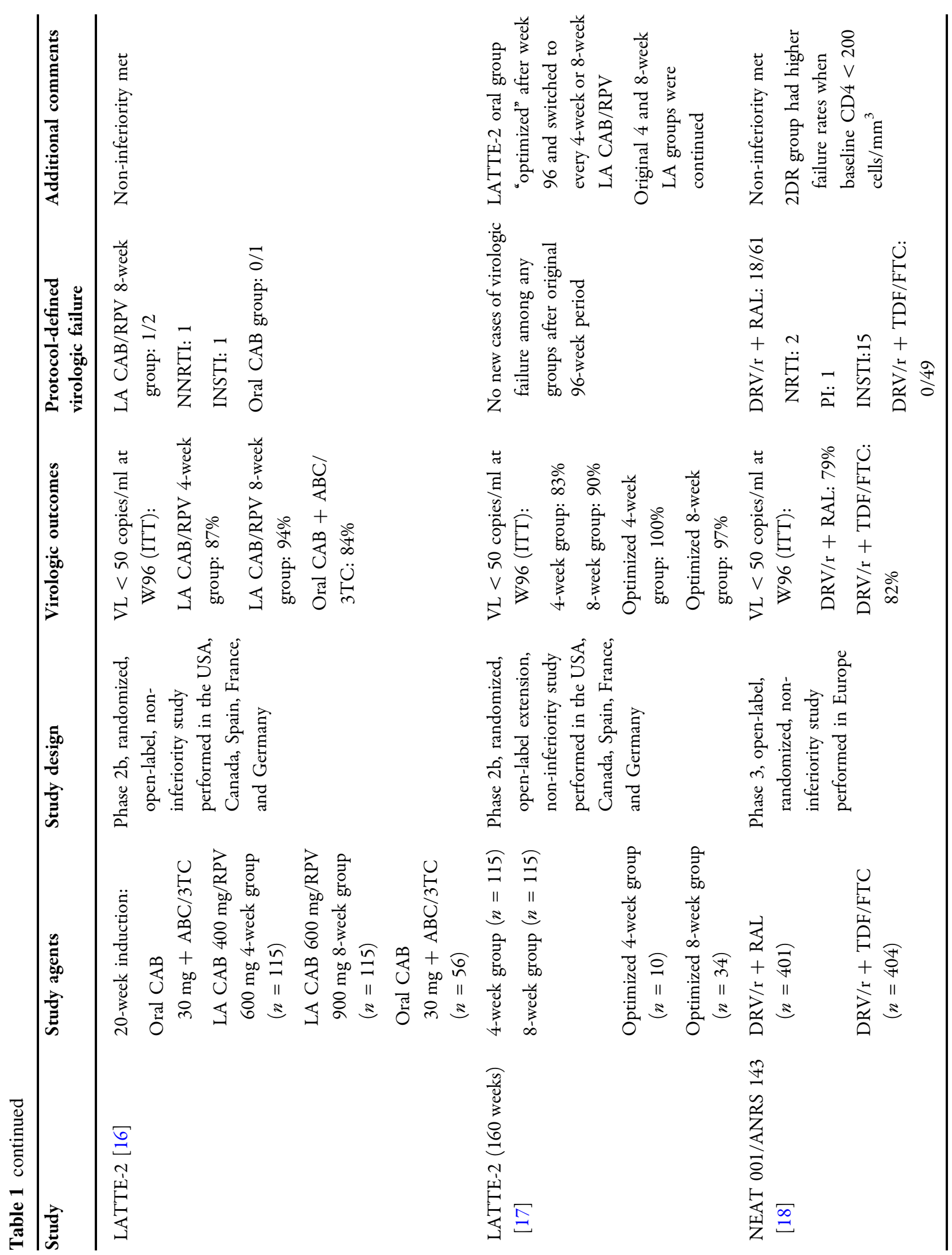




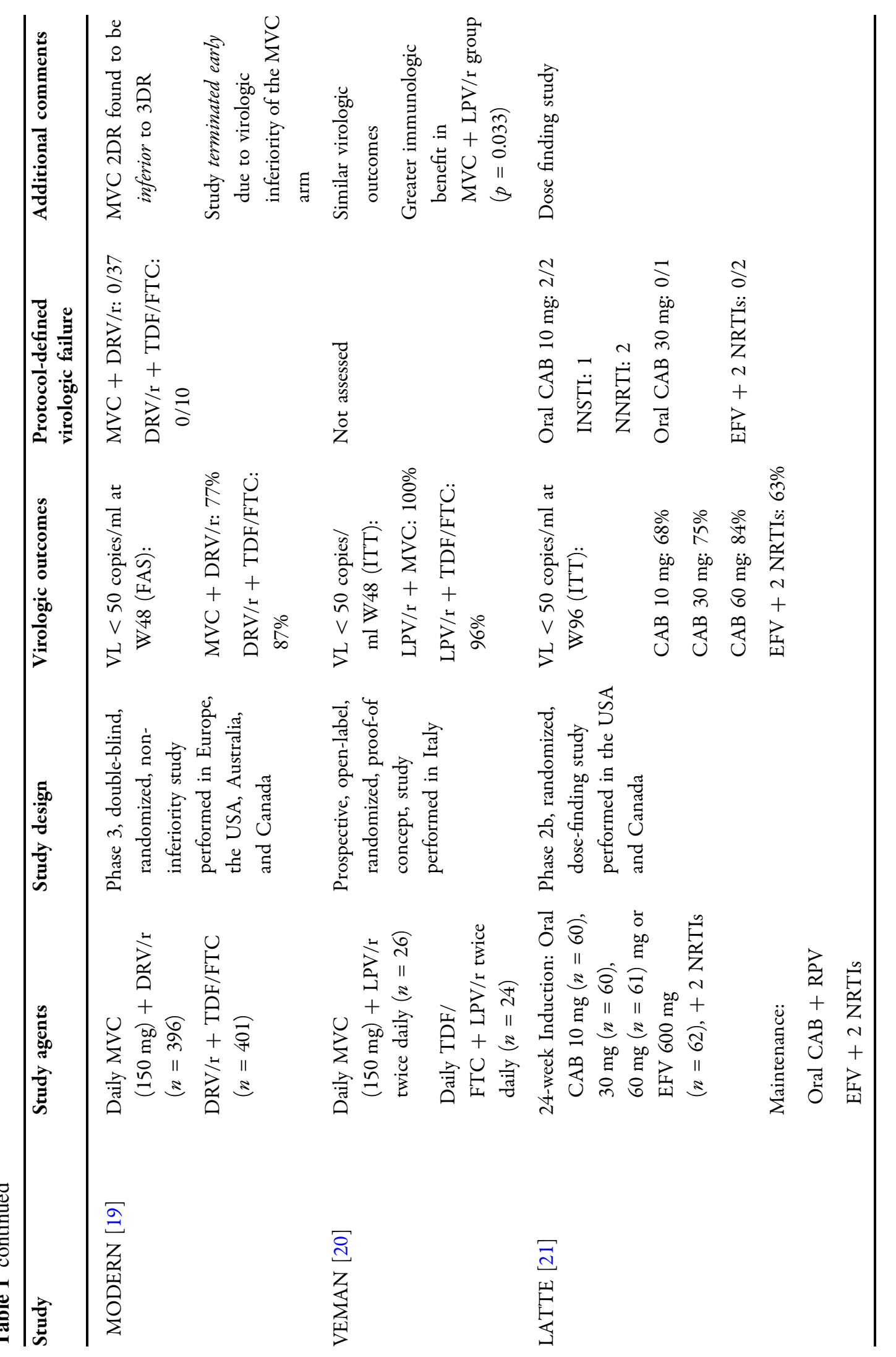




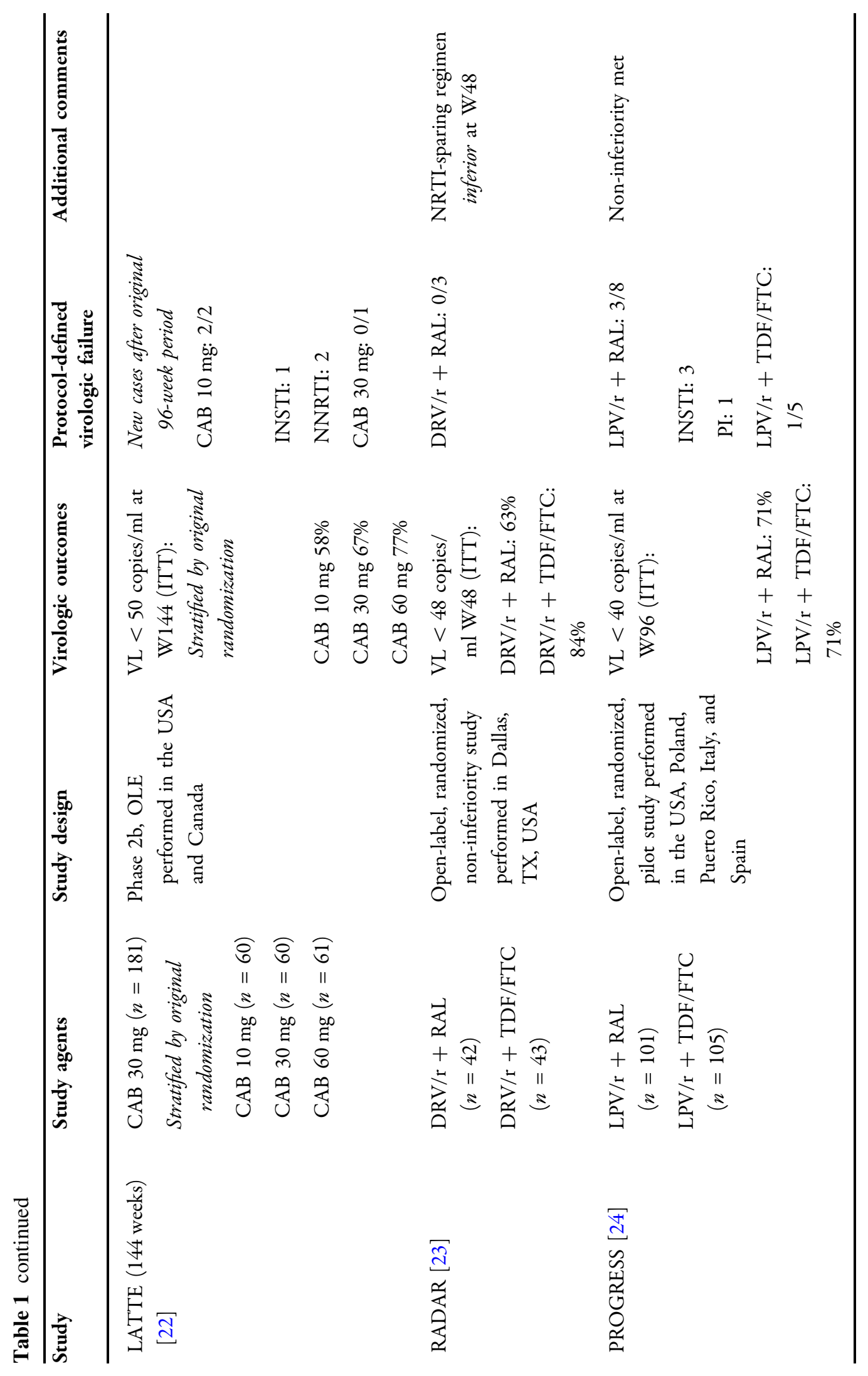




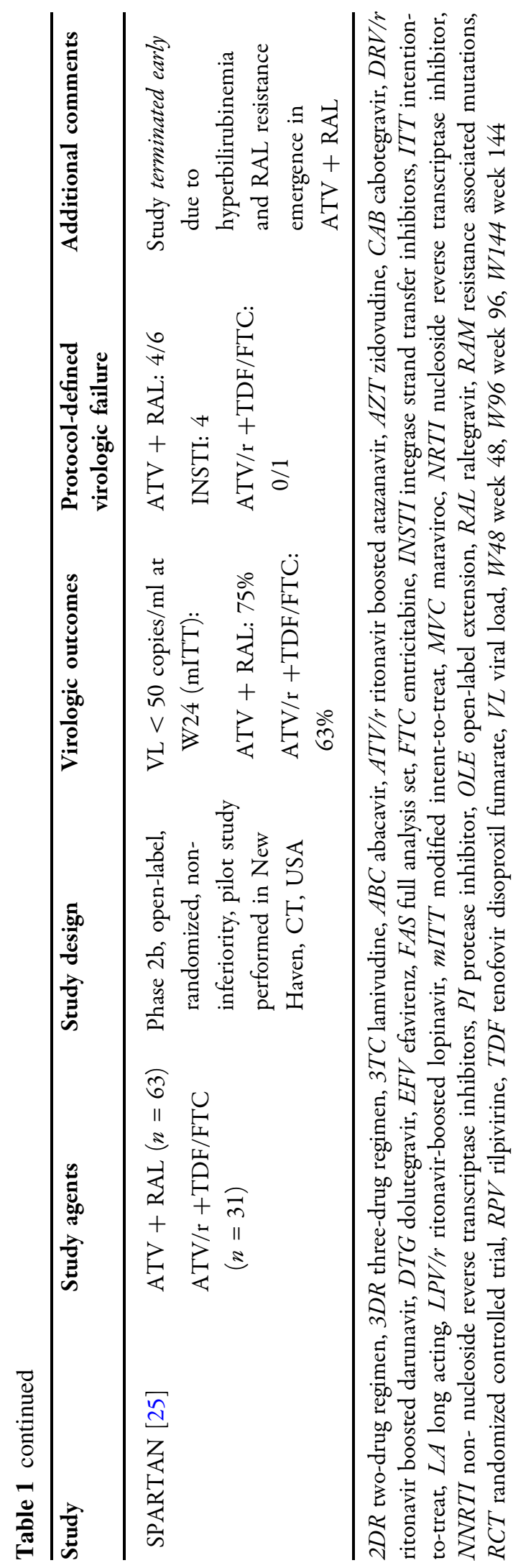

study of the same regimens achieved non-inferiority at 96 weeks and found increased rates of resistance and treatment failure among patients with low CD4 counts (i.e., $<200$ cells $/ \mathrm{mm}^{3}$ ) or high baseline HIV-RNA viral loads (i.e., > 100,000 copies/ml) [18]. A single pilot study of RAL + unboosted atazanavir (ATV) failed to achieve non-inferiority and was terminated early because of high rates of resistance and toxicities emerging from the once-daily dosing regimen of ATV (SPARTAN) [25].

Three studies included the new INSTI being evaluated for approval, cabotegravir (CAB), plus the NNRTI RPV [15-17, 21, 22]. Of those studies, one was a dose-finding study for oral formulations and two were of the novel, longacting (LA) formulations. Data from LATTE demonstrated patients receiving oral $\mathrm{CAB}$ $30 \mathrm{mg}+\mathrm{RPV} 25 \mathrm{mg}$ daily experienced high rates of viral suppression at week 96 (75\%) and tolerated the regimen well without any development of resistance [21, 22]. Based on these results, the $\mathrm{CAB} 30 \mathrm{mg}$ once-daily dose was selected for continued evaluation. LATTE-2 utilized the $30 \mathrm{mg} \mathrm{CAB}$ dose as part of a 20-week, 3DR induction phase, which was followed by a novel 2DR of LA, injectable formulation of $\mathrm{CAB}$ and RPV given once every 4 or 8 weeks [16, 17]. Non-inferiority at 96 weeks was achieved in both groups relative to the continuation of the induction phase 3DR, with minimal resistance emerging in the LA 8-week group. The LA formulations dosed every 4 and 8 weeks were well tolerated, with frequent, yet expected, mild injection site reactions. Patient survey data also demonstrated increases in treatment satisfaction and preference for the LA 2DR formulation. Comprehensive data from the FLAIR (treatment-naïve) and ATLAS (treatmentexperienced) studies demonstrated virologic suppression in $93.1 \%$ in the LA CAB/RPV arm and $94.4 \%$ in the current antiretroviral regimen (CAR) arm, which was 3DR at week 48 and met non-inferiority. Although seven participants in the FLAIR study experienced confirmed virologic failure (CVF), three in the 2DR arm and four in the 3DR arm, three out of four in the CAR/3DR arm did not have treatment-emergent resistance (resistance-associated mutation (RAM) was not reported) [15]. Interestingly, 


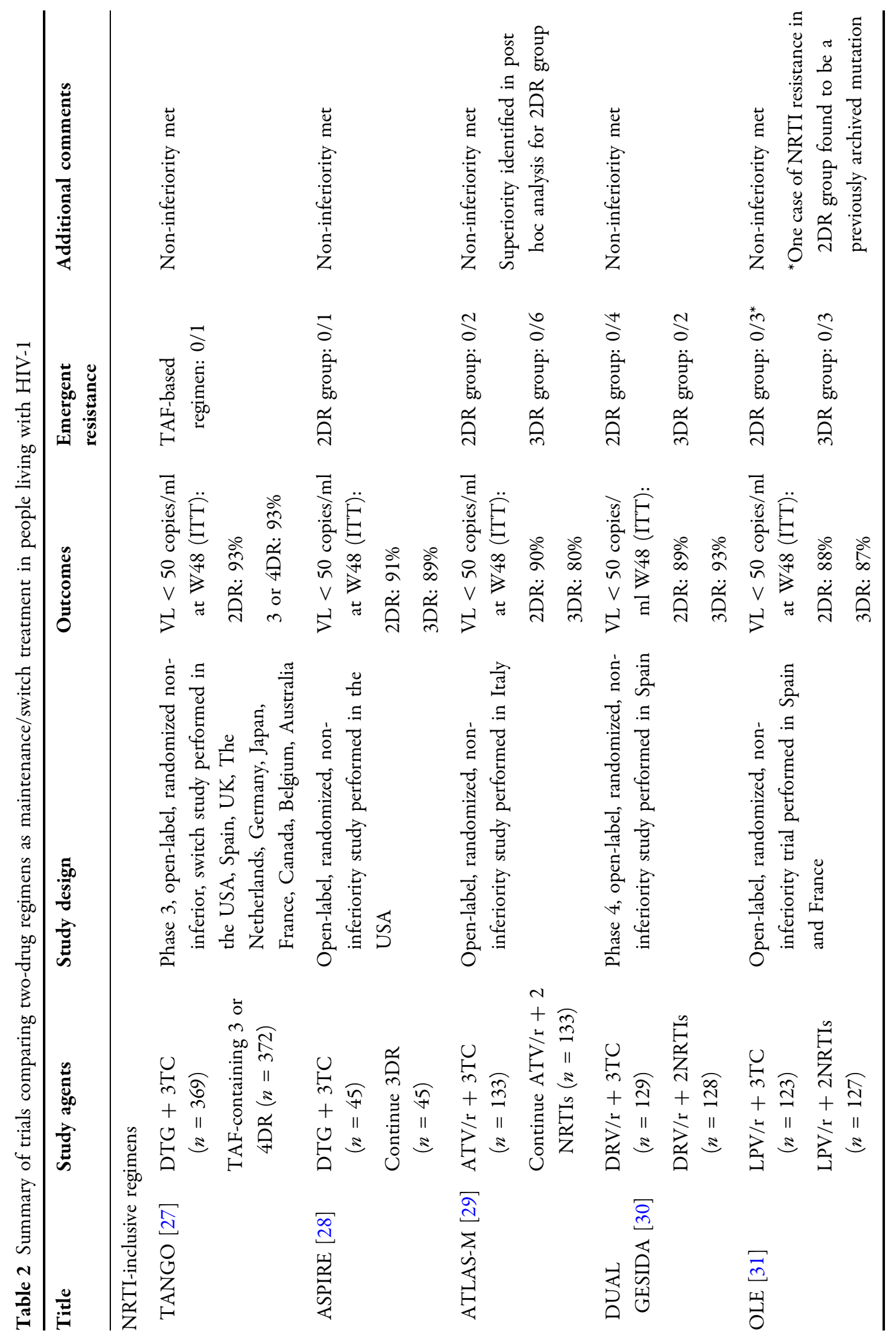




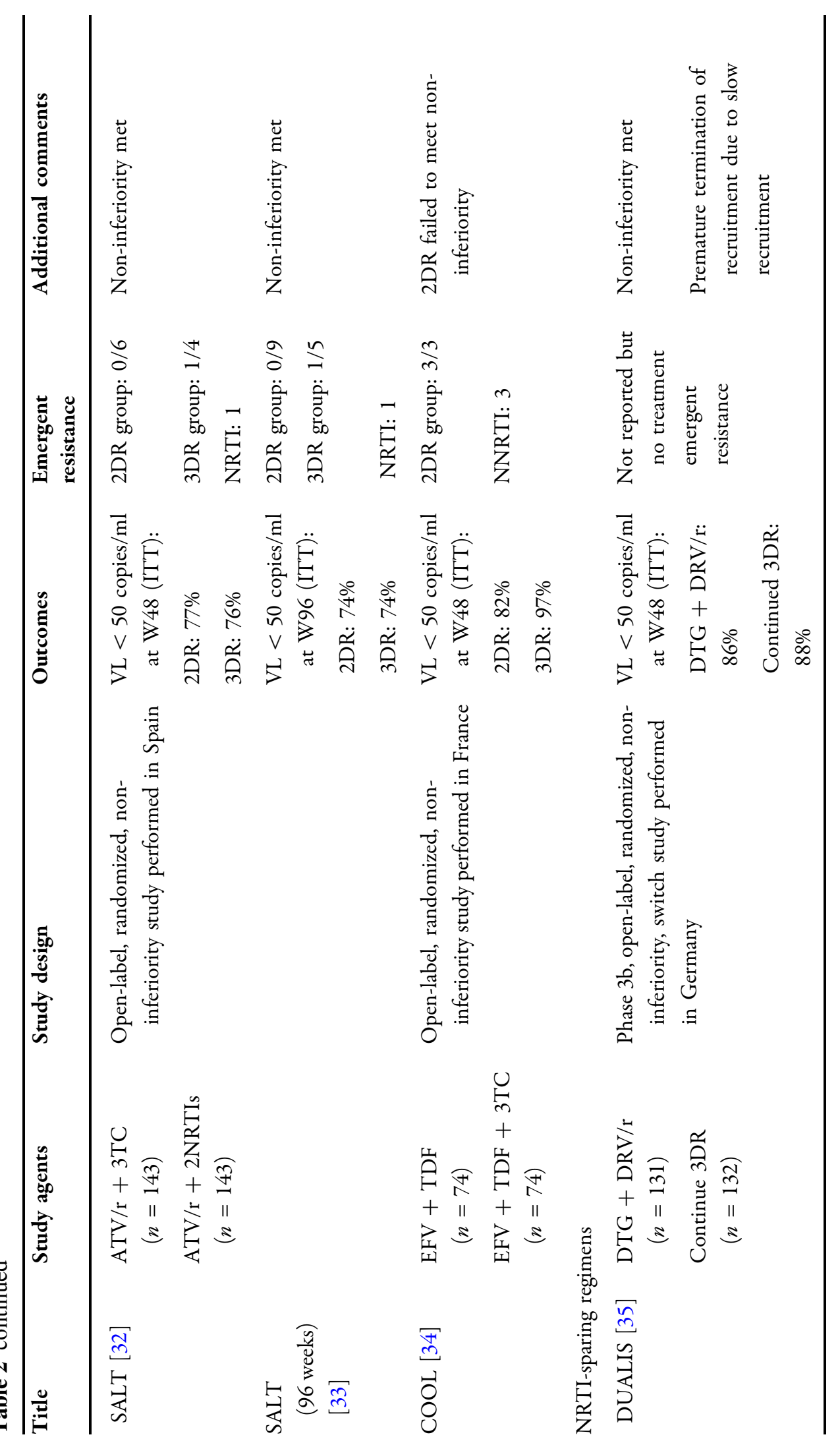




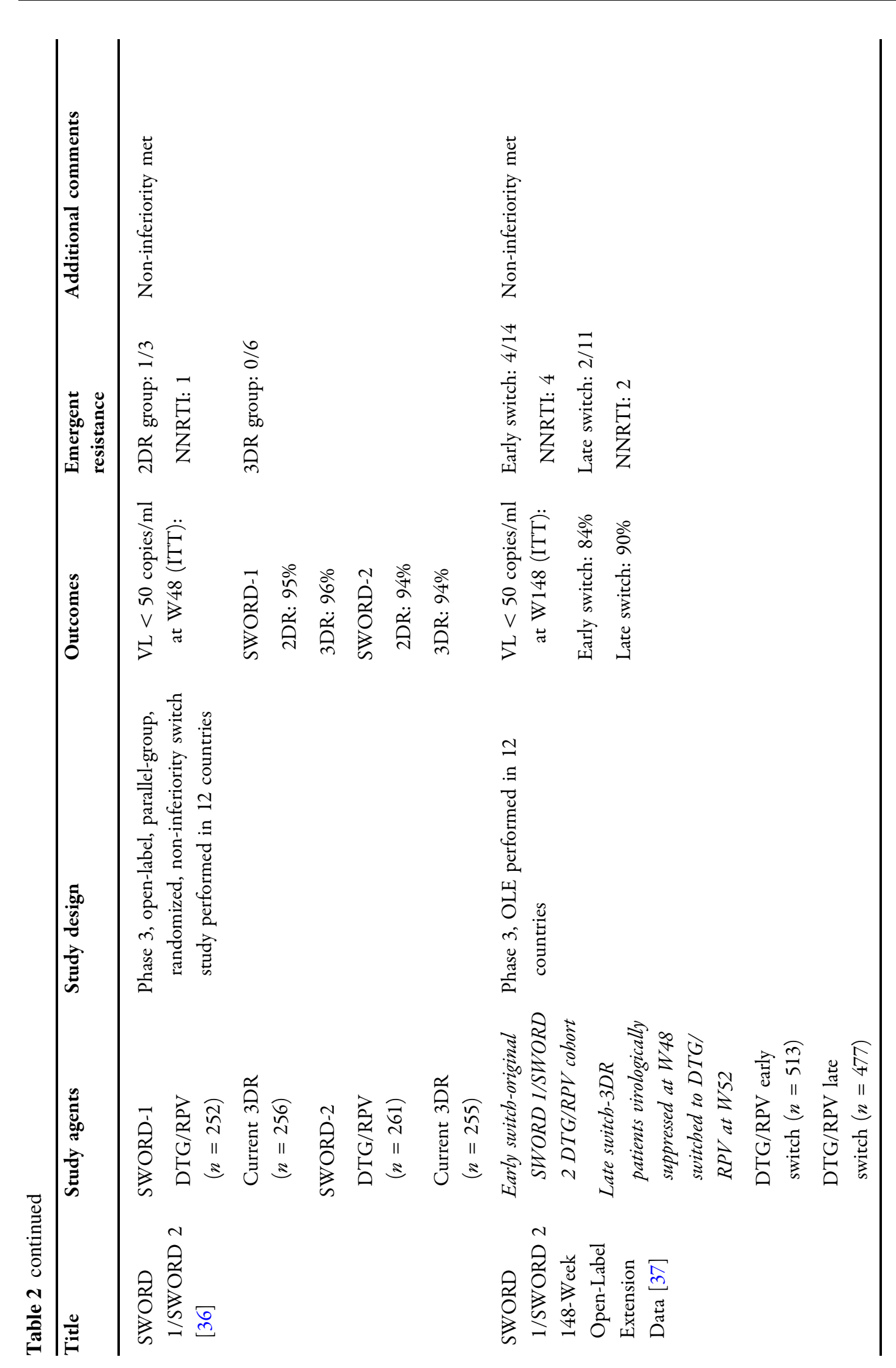




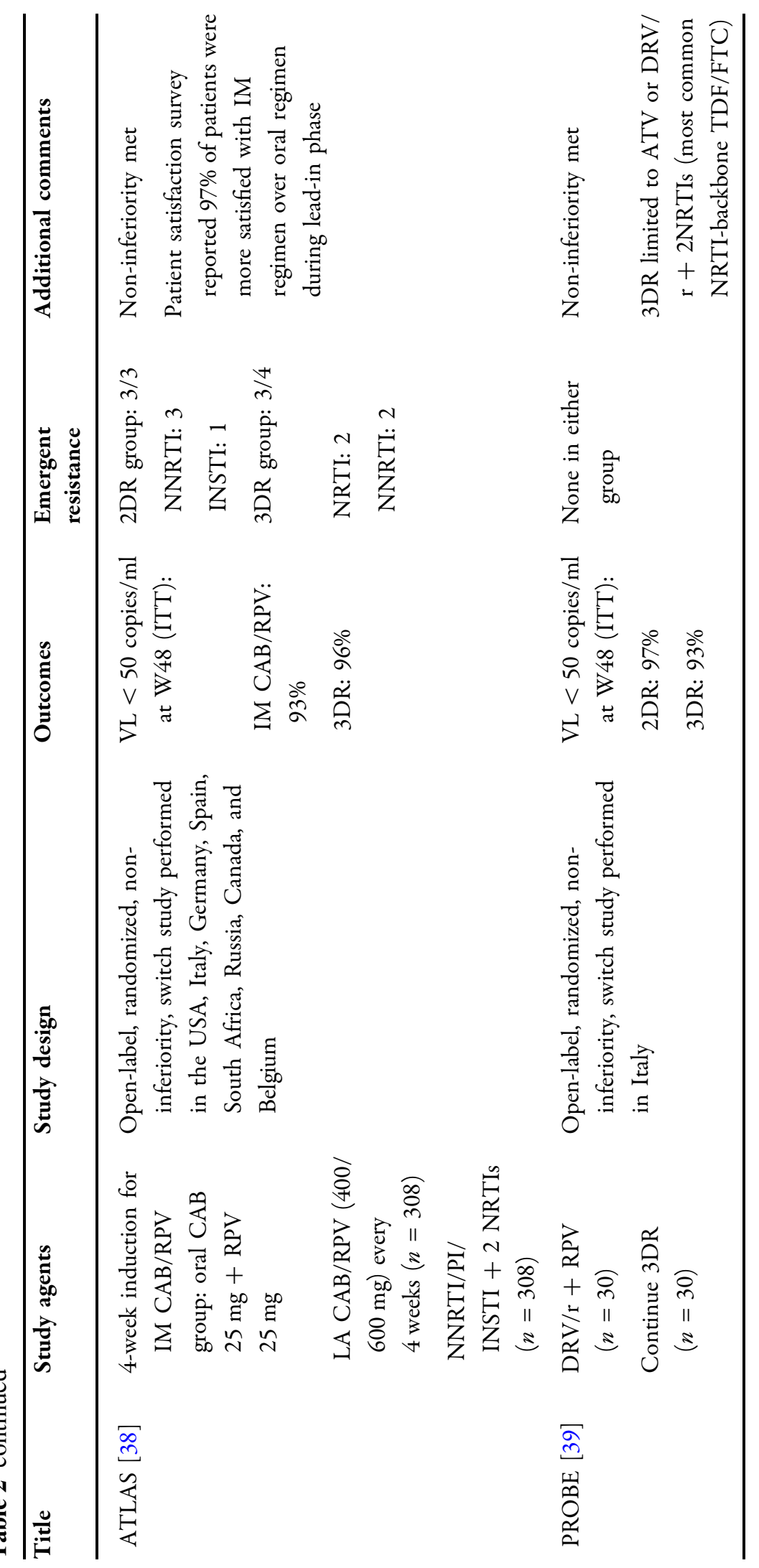




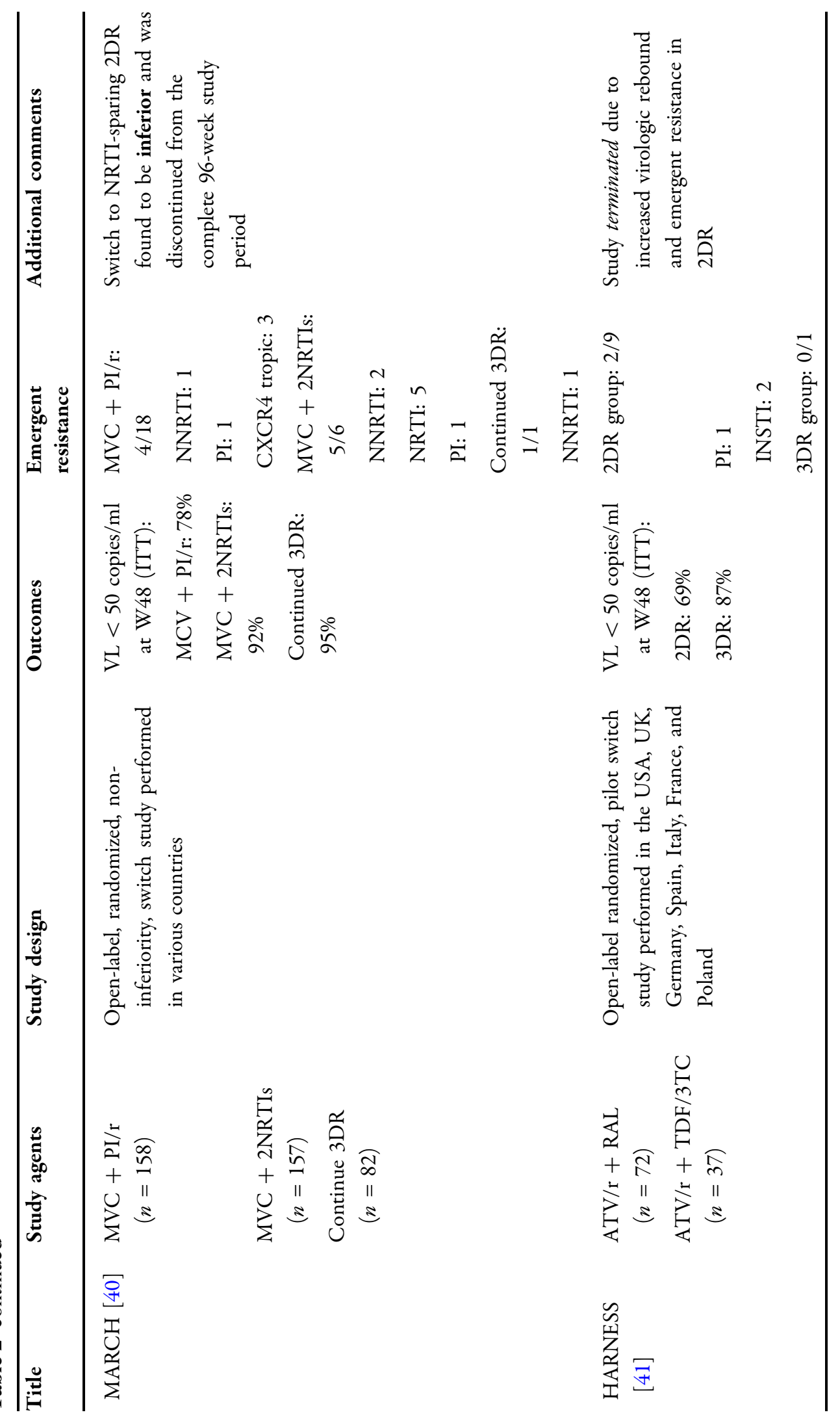




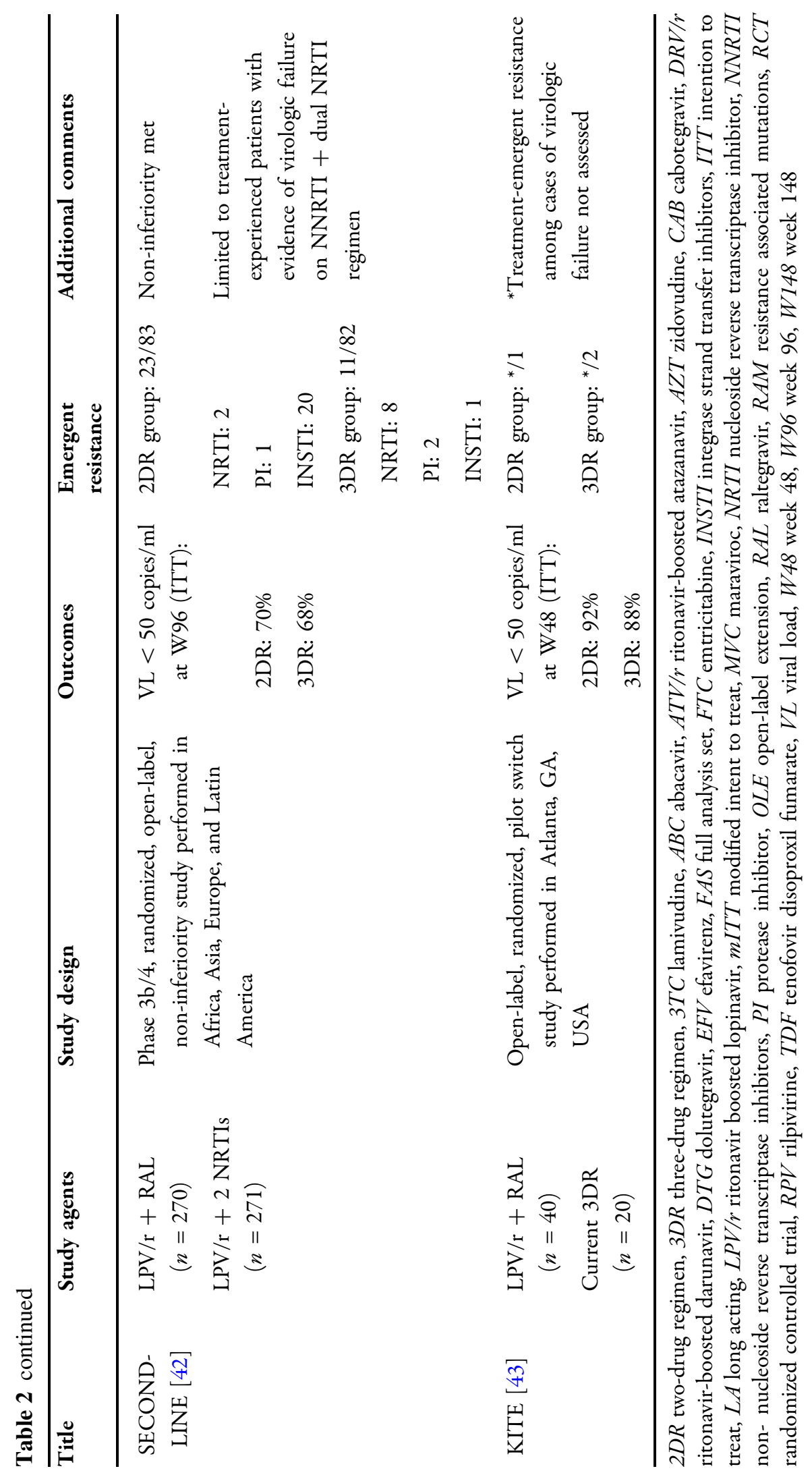


participants who developed resistance in the LA $\mathrm{CAB} / \mathrm{RPV}$ group were all from Russia and were found to have the polymorphism L74I at baseline, which is associated with INSTI resistance.

\section{Maintenance/Switch Treatment Population}

\section{NRTI Inclusive}

Seven non-inferiority studies of NRTI-inclusive 2DRs in treatment-experienced populations were identified (Table 2) [27-34]. The COOL study evaluating EFV + TDF vs. EFV + TDF/3TC failed to achieve non-inferiority [34]. The remaining six studies each included the NRTI 3TC as part of the 2DR and met non-inferiority at 48 weeks without any cases of emergent resistance. Four of the studies included a boosted PI + 3TC (two studied ATV/r, one LPV/r, and one DRV/r) [29-33], while two studies included DTG + 3TC [27, 28]. While safety outcomes from the studies were consistent with previous findings, studies including ATV produced more cases of hyperbilirubinemia, scleral icterus, and elevations in liver function tests (LFTs) and bilirubin $[29,31,32]$, while the study including LPV/r experienced more frequent GI upset [31].

\section{NRTI Sparing}

Eight studies were identified on NRTI-sparing regimens used in treatment-experienced patients (Table 2) [35-43]. All but one study evaluated patients that were virologically suppressed and on a stable ART regimen prior to randomization, although they varied in definition and required duration of viral suppression. Of the six non-inferiority studies, including a variety of $2 \mathrm{DR}$ combinations, non-inferiority was met in five studies [35-39]. The switch strategy of replacing a dual NRTI backbone with MVC in a boosted-PI regimen was found to be inferior to continuing a 3DR [40].

The SWORD studies and ATLAS studies included INSTI + NNRTI 2DRs [36-38]. SWORD 1 and 2 demonstrated the 2DR of DTG/RPV met the pre-specified non-inferiority criteria to standard 3DR [36]. The open-label extension of SWORD 1 and 2 also demonstrated durable efficacy in both early- and late-switch DTG/RPV groups [37]. There was one case of treatmentemergent NNRTI resistance among the 2DR arm in the first 48 weeks and five cases of NNRTI resistance in the early- and late-switch groups.

The ATLAS study demonstrated high rates of virologic suppression at 48 weeks in individuals with a suppressed viral load who were switched to a 4-week lead-in of oral CAB + RPV followed by a once-monthly injectable LA CAB/RPV regimen [38]. The study showed good tolerability, minimal emergence of treatment-related resistance, and high patient satisfaction of a oncemonthly injectable 2DR. ATLAS-2 $\mathrm{M}$ is an ongoing non-inferiority study evaluating LA $\mathrm{CAB} / \mathrm{RPV}$ administered every 8 weeks compared with every 4 weeks in virologically suppressed patients [44]. The study demonstrated LA CAB/ RPV given every 8 weeks was non-inferior to 4 week dosing through 48 weeks. One study utilized a PI + NNRTI 2DR, which showed favorable results. In a small proof-of-concept study DRV/r + RPV was found to be non-inferior and well tolerated without emergence of resistance [39].

Three studies included RAL + boosted PI [41-43]. A pilot study of $\mathrm{LPV} / \mathrm{r}+\mathrm{RAL}$ was shown to be a viable switch strategy out to 48 weeks [43]; however, a study of ATV/r + RAL was terminated early because of increased rates of virologic rebound and treatment-emergent resistance [41]. Another study included treatment-experienced patients with evidence of virologic failure on an NNRTI + dual NRTI regimen. The use of $\mathrm{LPV} / \mathrm{r}+\mathrm{RAL}$ as second-line therapy showed promising results relative to a 3DR containing $\mathrm{LPV} / \mathrm{r}+$ dual NRTIs out to 96 weeks [42].

\section{DISCUSSION}

In both treatment-naïve and -experienced populations, 3TC was the most commonly used NRTI in 2DRs that met non-inferiority. Although 3TC and FTC are viewed in clinical practice as therapeutically interchangeable cytidine analogs, FTC has yet to be studied in the context of NRTI-inclusive 2DRs. Two studies of NRTI-inclusive 2DRs containing TDF as 
opposed to the more robustly studied 3TC both failed to achieve non-inferiority $[11,34]$. Thus, the data to date, do not support the use of TDF as a single NRTI agent in a 2DR. With the more recent approval date, TAF remains virtually unstudied by comparison or inclusion in the 2DR space.

3TC in combination with boosted PIs has demonstrated efficacy in treatment-naïve individuals. In the ANDES and GARDEL studies, $3 \mathrm{TC}+\mathrm{DRV} / \mathrm{r}$ and $3 \mathrm{TC}+\mathrm{LPV} / \mathrm{r}$ were non-inferior to 3DRs $[12,13]$. Boosted PI and 3TC regimens have also been shown to be effective 2DRs in treatment-experienced populations. ATV/ $\mathrm{r}+3 \mathrm{TC}$ regimens have consistently met noninferiority, evidenced in both simplification from a previous ATV/r-based regimen and switching from a variety of PI and NNRTI-based 3DRs (ATLAS-M, SALT) [29, 32, 33]. There is limited evidence studying DRV in this population, although results from the DUAL-GESIDA study are promising [30]. These studies have not been associated with treatment-emergent resistance and have similar rates of viral suppression to comparator 3DRs. Although a boosted PI + $3 \mathrm{TC}$ may be an effective 2DR in treatmentnaïve and -experienced populations, PI-associated toxicities including metabolic disorders and laboratory abnormalities found even with the newer, more well-tolerated PIs such as DRV may be a limitation as well as significant drug interactions. These limitations may hinder the use of boosted PI + 3TC regimens in real-world settings.

The most promising NRTI-inclusive 2DR in patients initiating ART is currently DTG +3 TC. Recent guideline updates now recommend the co-formulated 2DR, DTG + 3TC, as a consideration in the treatment-naïve population with the exception of individuals with pre-treatment HIV RNA > 500,000 copies/ml, active hepatitis B virus (HBV) coinfection, or initiation of ART prior to the availability of HIV reverse transcriptase genotype or HBV testing [4, 5]. Results from the TANGO study were recently presented and demonstrated non-inferiority of DTG +3 TC compared with TAF-based 3DR in virologically suppressed patients $[4,5]$. Although not approved, switch data look promising for the use of DTG + 3TC in the treatment-experienced population. DTG/3TC demonstrated excellent virologic suppression without emergence of resistance in the treatment-experienced population [28], in line with the large body of evidence that was established by the GEMINI studies [10, 11]. More data are needed, but DTG + 3TC likely represents the most promising NRTI-inclusive 2DR in treatment-experienced patients, avoiding the issues of drug interactions and more severe side effect profiles of boosted PI + 3TC regimens. Recent 48-week data from the ANRS 167 LAMIDOL single-arm study further support switching to DTG/3TC in select, virologically suppressed patients [45]. Similarly, the DOLAM triple-armed study showed few cases of treatment failure among patients switched to DTG + 3TC as continuing current 3DR, without emergence of resistance [46].

In both treatment-naïve and -experienced populations, integrase inhibitors play a large role in NRTI-sparing 2DRs. Raltegravir was studied in many 2DRs as it was the first INSTI on the market $[18,24,25,42]$. However, data from randomized-controlled studies largely do not support any additional benefit from the use of a boosted PI with RAL since this regimen is quickly becoming obsolete given that RAL is a first-generation INSTI that is less potent than newer agents such as DTG and BIC. In addition, RAL suffers from issues of increased pill burden and cross-resistance with elvitegravir (EVG).

Dolutegravir has been studied in both NRTIinclusive and -sparing 2DRs. Unlike RAL, DTG has a long half-life and is efficacious in the presence of other INSTI resistance such as the Q148 pathway selected for with RAL or EVG. In NRTI-sparing 2DRs, it has been studied with both PIs and NNRTIs in mostly treatment-experienced populations. A regimen commonly considered in clinical practice consists of DRV/ $\mathrm{r}+$ DTG. This regimen combines two highly potent agents with high genetic barriers to resistance. This regimen is currently being investigated in treatment-experienced populations, as both a simplification strategy for virologically suppressed patients and a salvage therapy $[35,47,48]$. Recent data from a subanalysis of the DUALIS study met non-inferiority. At week $48,98.1 \%$ of patients $(n=113)$ in 
the observational TIVISTA study achieved virologic suppression $(<50$ copies $/ \mathrm{ml})$ despite 18 subjects experiencing reduced sensitivity to darunavir at baseline. No participant developed new drug resistance mutations throughout the study [49]. Additional observational studies have demonstrated similar results although $\mathrm{DRV} / \mathrm{r}$ and/or DTG was allowed twice daily [48] while another retrospective study demonstrated the feasibility of cobicistat-boosted DRV with DTG once daily in treatment-experienced patients [50].

Dolutegravir/RPV is the simplest option with the lowest pill burden for virologically suppressed PLWH, who do not have underlying resistance to either agent. Patients must be HBV-negative with demonstrated virologic suppression for at least 6 months with a CD4 count of $>200$ cells $/ \mathrm{mm}^{3}$. In addition, the ideal patient for this regimen must be able to take this regimen with a full meal and avoid proton pump inhibitors. SWORD 1/2 studies met noninferiority between DTG/RPV and 3DRs [36]. Long-term extension data have shown 2DR durability with minimal development of resistance [37]. Two observational studies of DTG/ RPV used in treatment-experienced patients have also demonstrated good efficacy among a real-world population [51, 52].

The most robust and emerging data of an INSTI containing 2DR are derived from studies of CAB and RPV as oral and LA intramuscular formulations. An open-label extension of LATTE demonstrated the durability of CAB $30 \mathrm{mg}+\mathrm{RPV}$ at 144 weeks [22]. It is important to note that patients studied in LATTE were required to undergo a 24 -week induction phase on oral $\mathrm{CAB}+2$ NRTIs prior to switching to a regimen of RPV as well as continuing their randomized $\mathrm{CAB}$ dose. In treatment-naïve patients, LA CAB/RPV has demonstrated the promising results in the achievement and durability of high rates of viral suppression, tolerability, and lack of significant treatmentemergent resistance. Furthermore, the LATTE-2 study also demonstrated durable viral suppression out to 160 weeks in the long-term extension data, with minimal emergence of resistance [17]. LA CAB/RPV represents a potential option in the future for PLWH.
However, as such therapies become available, a key consideration will be the necessity of an induction phase of oral $\mathrm{CAB}$ and how to best assess for initial safety and tolerability because of the long half-life and gap in care between injections. Furthermore, adherence during this induction phase will be crucial. Although safety and efficacy were demonstrated in clinical trials, the Food and Drug Administration denied approval of this co-formulated injectable secondary to chemistry manufacturing and controls [53].

Non-INSTI containing 2DRs that have shown positive results include a PI + NNRTI regimen. The 2DR of DRV/r + RPV appears promising from limited prospective data [39]. These results were confirmed with recent, real-world observational data, with viral suppression $<50$ copies $/ \mathrm{ml}$ achieved in $83 \%$ of patients (RIDAR) [54]. Several drawbacks of this regimen include the lack of a STR, increased adverse effects seen with PIs, and an increased potential for drugdrug interactions.

Although MVC was initially approved for management of resistant HIV virus, its recommended twice-daily dosing and numerous drug interactions leave it as an alternative treatment option. In addition, patients need to have a CCR5-tropic virus prior to initiation of therapy. Furthermore, maraviroc 2DRs in combination with PIs were shown to be inferior to 3DRs in studies in both treatment-naïve and -experienced populations.

\section{The "Ideal" 2DR}

Antiretroviral selection is key to ensuring a patient's success. Modern regimens achieve rapid viral suppression. However, the issue remains of the long-term durability of a regimen in maintaining virologic suppression without the emergence of resistance, particularly when considering incomplete adherence to an ART regimen. The optimal 2DR will have a low pill burden, high efficacy, minimal toxicity, reduced cost, and a high genetic barrier to resistance in both treatment-naïve and -experienced populations. Furthermore, as PLWH are living longer, it would also be beneficial for the 
ideal 2DR to have limited drug-drug interactions and not require renal or hepatic dose adjustments.

When considering 2DRs as initial or switch therapy, it is essential to assess for baseline and transmitted drug resistance. A major concern of a 2DR containing a single NRTI agent is the potential for the emergence of NRTI RAMs such as M184I/V, which greatly reduces susceptibility to 3TC and FTC. Recent data demonstrate effective use of 3TC as a single NRTI in a 2DR, with minimal emergence of NRTI resistance, including M184I/V mutations, in cases of virologic failure $[10,11]$. It should be noted that no difference was observed in treatment-naïve or experienced 2DR studies in the development of M184I/V mutations. Another benefit of 3TCcontaining regimens is the high tolerability. By utilizing 3TC as a single NRTI backbone, and avoiding or removing the use of TDF, negative effects on renal function and bone biomarkers may be eliminated or ameliorated.

The role of INSTIs has revolutionized ART based on their rapid achievement of virologic suppression, enhanced tolerability, and reduced drug interaction potential compared with PIs and NNRTIs.

The first-generation INSTIs, RAL and EVG, often display cross-resistance and have a lower genetic barrier to resistance; however, DTG was developed as a second-generation INSTI with improved resistance profile and high efficacy. Dolutegravir has demonstrated efficacy in 2DRs that are both NRTI-inclusive and sparing. Although BIC also has a high genetic barrier to resistance and demonstrates similar safety and tolerability to DTG, it is only available as a coformulated single-tablet 3DR. Cabotegravir has been studied as a LA 2DR, in combination with $\mathrm{RPV}$, in both treatment-naïve and -experienced populations. This 2DR appears very promising, especially with its novel formulation; however, its approval is still pending based on formulation concerns.

The emergence of 2DRs as initial therapy in treatment-naïve individuals and as simplification strategies in treatment-experienced populations has become a reality and is creating a paradigm shift in the management of HIV. Dual therapies are largely prescribed as simplification strategies in European countries and is becoming more favorable in the USA. Unfortunately, most studies evaluated standard 3DRs that were considered first line at the time of the study initiation. More modern comparators should be included in 2DR studies since most of the available head-to-head, randomized clinical trial data include TDF-based regimens.

Although 2DRs are being utilized more frequently, their use may not be appropriate in all patient populations. Concerns exist about the use of 2DRs in PLWH who have low CD4 counts $\left(<200\right.$ cells $\left./ \mathrm{mm}^{3}\right)$ or high baseline viral loads $(<100,000$ or 500,000 copies $/ \mathrm{ml}$ based on study design) prior to drug initiation [10, 11, 18]. Subgroup analyses of failure rates in these populations are often limited because of the small sample size and often not attributed to true virologic failure. Most studies, with the exception of rescue/salvage therapy aside, screen for and exclude patients with underlying baseline resistance to the regimen that they will be initiated or switched to. Other populations in which 2DRs should be avoided include pregnant individuals and those with hepatitis $B$ coinfection.

While the overall picture of evidence on 2DRs remains mixed, it is important to note the significant changes in the HIV treatment landscape that have taken place over the last decade. Another question remains about how the data from switch studies are reported and applied to the management of HIV. Particularly in switch studies evaluating treatment-naïve and -experienced patients, is accepting "non-inferiority" enough? There has been a recent change in reporting outcomes for assessing the non-inferiority of HIV switch strategies. The US Food and Drug Administration recommends primary end points of switch trials to report the rate of virologic failure in each treatment group where non-inferiority would be a margin of $\leq 4 \%$ [55].

\section{CONCLUSION}

HIV treatment has evolved from a time where 2DRs were once considered a novel concept to current times where they are a reality. As 2DRs are reducing the number of medications 
required to manage $\mathrm{HIV}$, while maintaining durable efficacy, 3DR may no longer remain the standard of care but become the antiquated way of the past.

\section{ACKNOWLEDGEMENTS}

Funding. No funding or sponsorship was received for this study or publication of this article.

Authorship. All named authors meet the International Committee of Medical Journal Editors (ICMJE) criteria for authorship for this manuscript, take responsibility for the integrity of the work as a whole, and have given final approval for the version to be published.

Disclosures. Melissa Badowski, Sarah Perez, David Silva, and Andrea Lee have no disclosures to report.

Compliance with Ethics Guidelines. This article is based on previously conducted studies and does not involve any new studies of human or animal subjects performed by any of the authors.

Data Availability. Data sharing is not applicable to this article as no data sets were generated or analyzed during the current study.

Open Access. This article is licensed under a Creative Commons Attribution-NonCommercial 4.0 International License, which permits any non-commercial use, sharing, adaptation, distribution and reproduction in any medium or format, as long as you give appropriate credit to the original author(s) and the source, provide a link to the Creative Commons licence, and indicate if changes were made. The images or other third party material in this article are included in the article's Creative Commons licence, unless indicated otherwise in a credit line to the material. If material is not included in the article's Creative Commons licence and your intended use is not permitted by statutory regulation or exceeds the permitted use, you will need to obtain permission directly from the copyright holder. To view a copy of this licence, visit http://creativecommons.org/licenses/by$\mathrm{nc} / 4.0 /$.

\section{REFERENCES}

1. Broder $S$. The development of antiretroviral therapy and its impact on the HIV-1/AIDS pandemic. Antivir Res. 2010;85:1-18.

2. Pau AK, George JM. Antiretroviral therapy: current drugs. Infect Dis Clin N Am. 2014;28:371-402.

3. U.S. Department of Health and Human Services. Panel on Antiretroviral Guidelines for Adults and Adolescents. Guidelines for the use of antiretroviral agents in adults and adolescents living with HIV. http://aidsinfo.nih.gov/contentfiles/lvguidelines/ AdultandAdolescentGL.pdf. Accessed 23 Jan 2020.

4. US Department of Health and Human Services. What to start: initial combination regiments for the antiretroviral-naïve patient. https://aidsinfo.nih. gov/guidelines/brief-html/1/adult-and-adolescentarv/11/what-to-start. Accessed 23 Jan 2020.

5. European AIDS Clinical Society. EACS guidelines version 10.0 (Nov 2019). https://eacs.sanfordguide. com/. Accessed 23 Jan 2020.

6. Wijting I, Rokx C, Boucher C, et al. Dolutegravir as maintenance monotherapy for HIV (DOMONO): a phase 2, randomised non-inferiority trial. Lancet HIV. 2017;4:e547-54.

7. Hocqueloux L, Raffi F, Prazuck T, et al. Dolutegravir monotherapy versus dolutegravir/abacavir/lamivudine for virologically suppressed people living with chronic HIV infection: the randomized non-inferiority MONCAY trial. Clin Infect Dis. 2019;69: 1498-505.

8. Galli L, Spagnuolo V, Bigoloni A, et al. Atazanavir/ ritonavir monotherapy: 96 week efficacy, safety and bone mineral density from the MODAt randomized trial. J Antimicrob Chemother. 2016;71:1637-42.

9. Juluca [package insert]. Research Triangle Park, NC. ViiV Healthcare; 2019.

10. Cahn P, Madero JS, Arribas JR, et al. Dolutegravir plus lamivudine versus dolutegravir plus tenofovir disoproxil fumarate and emtricitabine in antiretroviral-naive adults with HIV-1 infection (GEMINI-1 and GEMINI-2): week 48 results from two multicentre, double-blind, randomised, non-inferiority, phase 3 trials. Lancet. 2019;393:143-55. 
11. Cahn, P et al. Durable efficacy of dolutegravir (DTG) plus lamivudine (3TC) in antiretroviral treatment-naïve adults with HIV-1 infection-96week results from the GEMINI studies. Presented at: 10th International AIDS Conference on HIV Science (IAS 2019); July 21-24, 2019, Mexico City, Mexico.

12. Figueroa MI, Sued OG, Gun AM, Belloso WH, Cecchini DM, Lopardo G. DRV/R plus 3TC for HIV-1 treatment naive patients: week 48 results of the ANDES study. Presented at: 2018 Conference on Retroviruses and Opportunistic Infections (CROI); March 4-7, 2018. Boston, MA, USA

13. Cahn P, Andrade-Villanueva J, Arribas JR, et al. Dual therapy with lopinavir and ritonavir plus lamivudine versus triple therapy with lopinavir and ritonavir plus two nucleoside reverse transcriptase inhibitors in antiretroviral-therapy-naive adults with HIV-1 infection: 48 week results of the randomised, open label, non-inferiority GARDEL trial. Lancet Infect Dis. 2014;14:572-80.

14. Pinola M, Lazzarin A, Antinori A, et al. Lopinavir/ ritonavir + tenofovir dual therapy versus lopinavir/ ritonavir-based triple therapy in HIV-infected antiretroviral naïve subjects: the Kalead Study. J Antivir Antiretrovir. 2010;2:56-62.

15. Orkin C, Arasteh K, Hernandez-Mora MG, et al. Long-acting cabotegravir + rilpivirine for HIV maintenance: FLAIR week 48 results. Presented at: 2019 CROI. March 4-7, 2019; Seattle, WA, USA

16. Margolis DA, Gonzalez-Garcia J, Stellbrink HJ, et al. Long-acting intramuscular cabotegravir and rilpivirine in adults with HIV-1 infection (LATTE-2): 96-week results of a randomised, open-label, phase 2b, non-inferiority trial. Lancet. 2017;390: 1499-510.

17. Margolis DA, et al. Safety, efficacy and durability of long-acting CAB and RPV as two drug IM maintenance therapy for HIV-1 infection: LATTE-2 week 160 results. Presented at: Glasgow HIV. October 28-31, 2018; Glasgow, UK.

18. Lambert-Niclot S, George EC, Pozniak A, et al. Antiretroviral resistance at virological failure in the NEAT 001/ANRS 143 trial: raltegravir plus darunavir/ritonavir or tenofovir/emtricitabine plus darunavir/ritonavir as first-line ART. J Antimicrob Chemother. 2016;71:1056-62.

19. Stellbrink HJ, Le Fevre E, Carr A, et al. Once-daily maraviroc versus tenofovir/emtricitabine each combined with darunavir/ritonavir for initial HIV-1 treatment. AIDS. 2016;30:1229-38.

20. Nozza S, Galli L, Antinori A, et al. Maraviroc $150 \mathrm{mg}$ daily plus lopinavir/ritonavir, a nucleoside/ nucleotide reverse transcriptase inhibitor-sparing regimen for HIV-infected naive patients: 48-week final results of VEMAN study. Clin Microbiol Infect. 2015;21(510):e1-9.

21. Margolis DA, Brinson CC, Smith GHR, et al. Cabotegravir plus rilpivirine, once a day, after induction with cabotegravir plus nucleoside reverse transcriptase inhibitors in antiretroviral-naive adults with HIV-1 infection (LATTE): a randomised, phase $2 b$, dose-ranging trial. Lancet Infect Dis. 2015;15:1145-55.

22. Margolis DA, Brinson C, Smith GH, et al. Long-term safety and efficacy of CAB and RPV as 2-drug oral maintenance therapy. Poster presented at: 2017 CROI; February 13-16, 2017; Seattle, WA, USA

23. Bedimo RJ, Drechsler $H$, Jain $M$, et al. The RADAR study: week 48 safety and efficacy of RAltegravir combined with boosted DARunavir compared to tenofovir/emtricitabine combined with boosted darunavir in antiretroviral-naive patients. Impact on bone health. PLoS One. 2014;9:e106221.

24. Reynes J, Trinh R, Pulido F, et al. Lopinavir/ritonavir combined with raltegravir or tenofovir/ emtricitabine in antiretroviral-naive subjects: 96-week results of the PROGRESS study. AIDS Res Hum Retroviruses. 2013;29:256-65.

25. Kozal MJ, Lupo S, Dejesus E, et al. A nucleoside- and ritonavir-sparing regimen containing atazanavir plus raltegravir in antiretroviral treatment-naïve HIV-infected patients: SPARTAN study results. HIV Clin Trials. 2012;13:119-30.

26. Saumoy M, Llibre JM, Terron A, et al. Short communication: maraviroc once-daily: experience in routine clinical practice. AIDS Res Hum Retrovir. 2017;33:29-32.

27. van Wyk J, Ajana F, Bisshop F, et al. Switching to DTG/3TC fixed-dose combination (FDC) is non-inferior to continuing a TAF-based regimen in maintaining virologic suppression through 48 weeks (TANGO study). Presented at: IAS 2019; July 21-24, 2019; Mexico City, México.

28. Taiwo BO, Marconi VC, Berzins B, et al. Dolutegravir plus lamivudine maintains human immunodeficiency virus-1 suppression through week 48 in a pilot randomized trial. Clin Infect Dis. 2018;66: 1794-7.

29. Di Giambenedetto S, Fabbiani M, Quiros Roldan E, et al. Treatment simplification to atazanavir/ritonavir + lamivudine versus maintenance of atazanavir/ritonavir + two NRTIs in virologically suppressed HIV-1-infected patients: 48 week results from a randomized trial (ATLAS-M). J Antimicrob Chemother. 2017;72:1163-71. 
30. Pulido F, Ribera E, Lagarde M, et al. Dual therapy with darunavir and ritonavir plus lamivudine vs triple therapy with darunavir and ritonavir plus tenofovir disoproxil fumarate and emtricitabine or abacavir and lamivudine for maintenance of human immunodeficiency virus type 1 viral suppression: randomized, open-label, noninferiority DUAL-GESIDA 8014-RIS-EST45 trial. Clin Infect Dis. 2017;65(12):2112-8.

31. Arribas JR, Girard PM, Landman R, et al. Dual treatment with lopinavir-ritonavir plus lamivudine versus triple treatment with lopinavir-ritonavir plus lamivudine or emtricitabine and a second nucleos(t)ide reverse transcriptase inhibitor for maintenance of HIV-1 viral suppression (OLE): a randomised, open-label, non-inferiority trial. Lancet Infect Dis. 2015;15:785-92.

32. Perez-Molina JA, Rubio R, Rivero A, et al. Dual treatment with atazanavir-ritonavir plus lamivudine versus triple treatment with atazanavir-ritonavir plus two nucleos(t)ides in virologically stable patients with HIV-1 (SALT): 48 week results from a randomised, open-label, non-inferiority trial. Lancet Infect Dis. 2015;15:775-84.

33. Perez-Molina JA, Rubio R, Rivero A, et al. Simplification to dual therapy (atazanavir/ritonavir + lamivudine) versus standard triple therapy [atazanavir/ritonavir + two nucleos(t)ides] in virologically stable patients on antiretroviral therapy: 96 week results from an open-label, non-inferiority, randomized clinical trial (SALT study). J Antimicrob Chemother. 2017;72:246-53.

34. Girard PM, Cabié A, Michelet C, et al. A randomized trial of two-drug versus three-drug tenofovir-containing maintenance regimens in virologically controlled HIV-1 patients. J Antimicrob Chemother. 2009;64:126-34.

35. Spinner CD, Kummerle T, Schneider J, et al. A switch to dolutegravir in combination with boosted darunavir is safe and effective in suppressed patients with HIV - a subanalysis of the DUALIS study. Presented at: IAS 2019: Conference on HIV Pathogenesis Treatment and Prevention; July 21-24, 2019; Mexico City, Mexico.

36. Llibre JM, Hung CC, Brinson C, et al. Efficacy, safety, and tolerability of dolutegravir-rilpivirine for the maintenance of virological suppression in adults with HIV-1: phase 3, randomised, non-inferiority SWORD-1 and SWORD-2 studies. Lancet. 2018;391:839-49.

37. van Wyk J, Orkin C, Rubio R, et al. Durable suppression and low rate of virologic failures 3 years after switch to DTG + RPV 2DRug Regimen: SWORD 1 and 2 Studies. Presented at: 25th Annual
Conference of the British HIV Association; April 2-5, 2019; Bournemouth, UK.

38. Swindells S, Andrade-Villanueva JF, Richmond GJ, et al. Long-acting cabotegravir rilpivirine maintenance therapy: ATLAS week 48 results. Presented at: 2019 CROI; March 4-7, 2019; Seattle, WA, USA

39. Maggiolo F, Di Filippo E, Valenti D, Serna Ortega PA, Callegaro A. NRTI sparing therapy in virologically controlled HIV-1 infected subjects: results of a controlled, randomized trial (Probe). J Acquir Immune Defic Syndr. 2016;72:46-51.

40. Pett SL, Amin J, Horban A, et al. Maraviroc, as a switch option, in HIV-1-infected individuals with stable, well-controlled HIV replication and R5-tropic virus on their first nucleoside/nucleotide reverse transcriptase inhibitor plus ritonavir-boosted protease inhibitor regimen: week 48 results of the randomized, Multicenter MARCH Study. Clin Infect Dis. 2016;63:122-32.

41. van Lunzen J, Pozniak A, Gatell JM, et al. Brief report: switch to ritonavir-boosted atazanavir plus raltegravir in virologically suppressed patients with HIV-1 infection: a randomized pilot study. J Acquir Immune Defic Syndr. 2016;71:538-43.

42. Amin J, Boyd MA, Kumarasamy N, et al. Raltegravir non-inferior to nucleoside based regimens in second-line therapy with lopinavir/ritonavir over 96 weeks: a randomised open label study for the treatment of HIV-1 infection. PLoS One. 2015;10: e0118228.

43. Ofotokun I, Sheth AN, Sanford SE, et al. A switch in therapy to a reverse transcriptase inhibitor sparing combination of lopinavir/ritonavir and raltegravir in virologically suppressed HIV-infected patients: a pilot randomized trial to assess efficacy and safety profile: the KITE study. AIDS Res Hum Retrovir. 2012;28:1196-206.

44. ViiV Healthcare. ViiV Healthcare reports positive phase III study results of investigational, long-acting, injectable HIV-treatment regimen administered every 2 months. https://viivhealthcare.com/ en-gb/media/press-releases/2019/august/viivhealthcare-reports-positive-phase-iii-study-resultsof-inve/. Accessed 6 Dec 2019.

45. Joly V, Burdet C, Landman R, et al. Dolutegravir and lamivudine maintenance therapy in HIV-1 virologically suppressed patients: results of the ANRS 167 trial (LAMIDOL). J Antimicrob Chemother. 2019;74:739-45.

46. Blanco JL, Rojas J, Paredes R, et al. Dolutegravirbased maintenance monotherapy versus dual therapy with lamivudine: a planned 24 week analysis of 
the DOLAM randomized clinical trial. J Antimicrob Chemother. 2018;73:1965-71.

47. Capetti AF, Sterrantino G, Cossu MV, Cenderello G, Cattelan AM, De Socio GV, Rusconi S, Riccardi N, Baldin GM, Cima S, Niero FP, Rizzardini G, Sasset L. Salvage therapy or simplification of salvage regimens with dolutegravir plus ritonavir-boosted darunavir dual therapy in highly cART-experienced subjects: an Italian cohort. Antivir Ther. 2017;22: 257-62.

48. Capetti AF, Cossu MV, Orofino G, Sterrantino G, Cenderello G, De Socio GV, Cattelan AM, Soria A, Rusconi S, Riccardi N, Baldin GM, Niero FP, Barbarini G, Rizzardini G. A dual regimen of ritonavir/darunavir plus dolutegravir for rescue or simplification of rescue therapy: 48 weeks' observational data. BMC Infect Dis. 2017;17:658.

49. Sterrantino G. Dolutegravir plus ritonavir-boosted darunavir in highly cART-experienced subjects. http://regist2.virology-education.com/2016/14EU/ 05_Sterrantino.pdf. Accessed 14 Jan 2020.

50. Lee SA, Kim SW, Chang HH, et al. Effectiveness, safety, and tolerability of a switch to dual therapy with dolutegravir plus cobicistat-boosted darunavir in treatment-experienced patients with human immunodeficiency virus. Infect Chemother. 2018;50:252-62.
51. Gantner P, Cuzin L, Allavena C, et al. Efficacy and safety of dolutegravir and rilpivirine dual therapy as a simplification strategy: a cohort study. HIV Med. 2017;18:704-8.

52. Capetti AF, Cossu MV, Sterrantino G, et al. Dolutegravir plus rilpivirine as a switch option in cART-experienced patients: 96-week data. Ann Pharmacother. 2018;52:740-6.

53. ViiV Healthcare. ViiV Healthcare receives complete response letter from US FDA for use of investigational cabotegravir and rilpivirine long-acting regimen in the treatment of HIV. https:// viivhealthcare.com/en-gb/media/press-releases/ 2019/december/complete-response-letter-from-usfda/. Accessed 30 Dec 2019.

54. Pasquau J, De Jesus SE, Arazo P, et al. Effectiveness and safety of dual therapy with rilpivirine and boosted darunavir in treatment-experienced patients with advanced HIV infection: a preliminary 24 week analysis (RIDAR study). BMC Infect Dis. 2019;19:207.

55. US Food and Drug Administration. Human immunodeficiency virus-1 infection: developing antiretroviral drugs for treatment. https://www.fda. gov/regulatory-information/search-fda-guidancedocuments/human-immunodeficiency-virus-1infection-developing-antiretroviral-drugstreatment. Accessed 24 Jan 2020. 